\title{
Current Endocardial Approaches for Left Atrial Appendage Closure
}

Domenico G Della Rocca, ${ }^{1}$ Armando Del Prete, ${ }^{2}$ Luigi Di Biase,${ }^{1,3,-6}$ Rodney P Horton, ${ }^{1}$ Amin Al-Ahmad, ${ }^{1}$ Mohamed Bassiouny, ${ }^{1}$ Sanghamitra Mohanty, ${ }^{1}$ Chintan Trivedi, ${ }^{1}$ Jorge Romero, ${ }^{5}$ Carola Gianni, ${ }^{1}$ J David Burkhardt, ${ }^{1}$ G Joseph Gallinghouse, ${ }^{1}$ Javier E Sanchez, ${ }^{1}$ Francesco Versaci, ${ }^{2}$ Andrea Natale, ${ }^{1,3,4,7-9}$

1. Texas Cardiac Arrhythmia Institute, St. David's Medical Center, Austin, TX, USA; 2. Division of Cardiology, S. Maria Goretti Hospital, Latina, Italy; 3. Department of Internal Medicine, Dell Medical School, University of Texas, Austin, TX, USA; 4. Department of Biomedical Engineering, Cockrell School of Engineering, University of Texas, Austin, TX, USA; 5. Arrhythmia Services, Department of Medicine, Montefiore Medical Center, Albert Einstein College of Medicine, Bronx, NY, USA; 6. Department of Clinical and Experimental Medicine, University of Foggia, Foggia, Italy; 7. Interventional Electrophysiology, Scripps Clinic, La Jolla, CA, USA; 8. Department of Cardiology, MetroHealth Medical Center, Case Western Reserve University School of Medicine, Cleveland, OH, USA; 9. Division of Cardiology, Stanford University, Stanford, CA, USA

DOI: https://doi.org/10.17925/EJAE.2019.5.1.40

$\mathrm{P}$ ercutaneous left atrial appendage (LAA) closure represents an attractive alternative to oral anticoagulation for stroke prophylaxis in patients with non-valvular atrial fibrillation. A growing body of evidence on the Amplatzer ${ }^{\mathrm{TM}}$ Cardiac Plug, the Amplatzer ${ }^{\mathrm{TM}}$ Amulet Device and the Watchman ${ }^{\mathrm{T}}$ device appears to confirm the safety and effectiveness of device-based strategies for stroke prevention. However, several questions still remain unanswered, mainly regarding patient and device selection, and post-procedural antithrombotic treatment. The present review summarises the rationale for LAA closure and presents the current device landscape for percutaneous LAA occlusion.

\section{Keywords}

Left atrial appendage, atrial fibrillation, device, stroke, oral anticoagulation

Disclosures: Luigi Di Biase is a consultant for Biosense Webster, Boston Scientific, Stereotaxis and St Jude Medical, and has received speaker honoraria from Medtronic, Atricure, EPiEP, and Biotronik. Andrea Natale has received speaker honoraria from Boston Scientific, Biosense Webster, St Jude Medical, Biotronik and Medtronic; and is a consultant for Biosense Webster, St Jude Medical and Janssen. Domenico G Della Rocca, Armando Del Prete, Rodney P Horton, Amin Al-Ahmad, Mohamed Bassiouny, Sanghamitra Mohanty, Chintan Trivedi, Jorge Romero, Carola Gianni, J David Burkhardt, G Joseph Gallinghouse, Javier E Sanchez and Francesco Versaci have nothing to declare in relation to this article.

Contributors: Domenico G Della Rocca and Armando Del Prete contributed equally to this work.

Review Process: Double-blind peer review.

Compliance with Ethics: This study did

not involve any studies with human or animal subjects performed by any of the authors.

Authorship: The named authors meet the International Committee of Medical Journal Editors (ICMJE) criteria for authorship for this manuscript, take responsibility for the integrity of the work as a whole and have given final approval for the version to be published.

Received: 13 February 2019

Accepted: 1 April 2019

Citation: European Journal of Arrhythmia \& Electrophysiology. 2019;5(1):40-6

Corresponding Author: Domenico Giovanni Della Rocca, Texas Cardiac Arrhythmia Institute at St. David's Medical Center, 3000 North 1-35, Suite 720, Austin, Texas 78705, USA. E: domenicodellarocca@hotmail.it

Support: No funding was received for

the publication of this article.
Atrial fibrillation (AF) is the most commonly encountered sustained arrhythmia and its prevalence and incidence are on the rise as the aging of the population increases. ${ }^{1} \mathrm{AF}$ has been described as an emerging epidemic of cardiovascular disease, with significant effects on estimated disability and mortality, and is rapidly turning into a major public health concern. This may be partially explained by the ageing of the population, the improved survival of patients with coronary artery disease and heart failure, and finally the increased capacity of clinicians to diagnose it. ${ }^{2}$ The overall prevalence of AF in the general population is around $2 \%,{ }^{3}$ with elderly patients showing an even higher prevalence (approximately $5.0 \%$ in patients $>65$ years and up to $8.8 \%$ among those aged 80 or older).

AF causes relevant clinical morbidity and represents an independent risk factor for mortality. The Framingham Heart Study, a longitudinal study conducted on 5,209 American adults for 40 years, demonstrated that $\mathrm{AF}$ predicts an increase in mortality with an odds ratio (OR) of 1.5 ( $95 \%$ confidence interval $[\mathrm{Cl}], 1.2-1.8)$ in men and $1.9(95 \% \mathrm{Cl}, 1.5-2.2)$ in women. ${ }^{4}$ The Marshfield Epidemiologic Study Area (MESA) showed a greater mortality among 577 patients with either AF or atrial flutter, when compared with controls, with a hazard ratio (HR) of $2.5(95 \% \mathrm{Cl}, 1.9-3.3)$ over a mean of 3.6 years of follow-up. ${ }^{5}$

$\mathrm{AF}$ is strongly associated with cardiovascular disease, acting as an independent risk factor for stroke, heart failure and mortality. Cardioembolic stroke accounts for $20 \%$ of all ischaemic strokes and in $90 \%$ of cases is associated with $A F$, regardless of the type of $A F^{2}{ }^{2}$

\section{Atrial fibrillation, left atrial appendage and stroke risk}

Stroke is a major cause of morbidity and mortality in the elderly, as well as one of the most common causes of disability in patients with AF. In the Framingham Heart Study, the annual risk of stroke attributable to AF was found to be around 1.5\% among patients aged 50 to 59 years, rising up to $23.5 \%$ among those older than 80 years during a follow-up period of 34 years. ${ }^{6}$ Stroke patients with AF also display a twofold risk of mortality and a threefold risk of recurrent stroke within 1 year compared to patients without AF.? Additionally, AF-related strokes tend to have higher mortality rates and be more incapacitating; therefore they lead to substantially higher healthcare costs than those not related to AF. ${ }^{8}$ In 2005, the total cost of AF care in the USA amounted to $\$ 6.65$ billion. ${ }^{9}$ Nearly two-thirds of the total economic burden of AF is represented by direct and indirect costs associated with hospitalisation, as well as the monitoring of pharmacological treatment and its adverse events. ${ }^{9}$ For these reasons, the use of new treatment strategies could help address the predicted epidemic of AF and its associated events, containing the estimated rise in healthcare costs. Stroke prevention strategies are of the utmost importance in AF management, 
and account for a significant proportion of health expenditure. The left atrial appendage (LAA) has been demonstrated to be the source of more than $90 \%$ of thrombi among patients with non-valvular AF presenting with stroke in whom a thrombus could be identified. ${ }^{10}$

The LAA derives from the primordial left atrium, formed by the adsorption of the primordial pulmonary veins and their branches. ${ }^{11}$ In a normally functioning heart, with intact contractility and adequate blood flow within the LAA, the risk of thrombus formation in the appendage is extremely low. Thrombi are more likely to form in the case of impaired contractility, with subsequent reduced blood flow velocity and stasis. AF determines ineffective atrial contractility and reduced heart function, ${ }^{12,13}$ with secondary remodelling of the LAA. Therefore, the appendage becomes dilatated and acts as a static pouch where blood stagnates, predisposing to thrombus formation. ${ }^{12}$ LAA morphology has been extensively studied via cardiac computed tomography and magnetic resonance imaging. Four major morphologies have been described, with each subtype bearing a different cardioembolic risk. ${ }^{14}$ The prevalence of different LAA morphologies was 'cactus' in 30\% of cases, 'chicken wing' (48\%), 'windsock' (19\%) and 'cauliflower' (3\%). After adjusting for the CHADS2 score, gender and AF types in a multivariable logistic model, 'chicken wing' morphology was found to be $79 \%$ less likely to be associated with a history of stroke/transient ischaemic attack (TIA). When considering 'chicken wing' as the reference group, 'cactus' was 4.08 times ( $p=0.046)$, 'windsock' was 4.50 times ( $p=0.038)$, and 'cauliflower' was 8.00 times $(\mathrm{p}=0.056)$ more likely to have had a stroke/TIA.

\section{Thromboembolic prophylaxis}

The traditional strategy for stroke prevention in patients with $\mathrm{AF}$ is oral anticoagulation $(\mathrm{OAC})$, which provides a valuable protection against cerebral and peripheral thromboembolism. OAC therapy is currently recommended to reduce the risk of thromboembolic events in patients with AF with a CHA2DS2-VASC score $\geq 2$ in males and $\geq 3$ in females. OAC should be considered in male patients with CHA2DS2-VASC score $\geq 1$ and female patients with a score $\geq 2$. ${ }^{1}$

For over 60 years, vitamin $\mathrm{K}$ antagonists (VKAs) have represented the standard of care inpatients with non-valvular AF. Although VKAs are highly effective in thromboembolic prevention, providing a $64 \%$ reduction of cerebrovascular accidents, ${ }_{1}^{15}$ they are also responsible for an increased risk of bleeding, which can sometimes be fatal. ${ }^{16}$ VKAs cannot be offered to all patients; warfarin is in fact contraindicated in around $10 \%$ of patients with non-valvular $\mathrm{AF}^{17}{ }^{17}$ In recent years, novel oral anticoagulants (NOACS) have proven to be superior to VKAs in terms of safety and efficacy, providing an additional 19\% reduction of the combined risk of stroke and systemic embolic events and an additional $10 \%$ reduction of the overall risk of death. ${ }^{15}$ NOACs are also associated with lower rates of major or clinically relevant non-major bleeding events and provide a 52\% reduction in the rate of intracranial bleedings compared to warfarin..$^{18}$ Even though NOACs require less complicated management, with no need for frequent monitoring and less drug-drug or drug-food interactions, it has been reported that adherence to NOACs is still poor, with up to one out of five patients discontinuing anticoagulant therapy. ${ }^{18}$ The optimal treatment plan for patients with AF should be based on a balanced assessment of benefits (e.g. stroke prevention) and risks (e.g. bleeding events).

Of note, up to $40 \%$ of patients with AF do not receive appropriate anticoagulation due to poor pharmacological adherence or the presence of contraindications, and they are at high risk for stroke. ${ }^{19}$ With this in mind, non-pharmacological therapies have been proposed as an alternative for stroke prophylaxis to address this unfilled therapeutic gap. Specifically, mounting evidence has shown that LAA exclusion procedures are an appealing and effective option in patients who cannot take VKAs and NOACs. The 2019 Focused Update of the 2014 American Heart Association/American College of Cardiology/Heart Rhythm Society (AHA/ACC/HRS) Guideline for the Management of Patients with Atrial Fibrillation' recommends considering percutaneous LAA occlusion for stroke prevention in patients with AF at increased risk of stroke who have contraindications to long-term anticoagulation (recommendation class: Ilb, level of evidence: B). However, important differences exist between the US Food and Drug Administration (FDA) indications and the Centers for Medicare \& Medicaid Services (CMS) approval. In the FDA approval, LAA occlusion may be considered in patients with an appropriate rationale for a non-pharmacological alternative to OAC. In the CMS approval, the occlusion device may be taken into consideration only in patients who are unsuitable for long-term OAC and have a CHADS2 score $\geq 2$ or a CHA2DS2-VASC score $\geq 3$.

\section{Endocardial approaches for left atrial appendage occlusion} Watchman and Watchman FLX left atrial appendage devices

The Watchman ${ }^{\mathrm{TM}}$ device (Boston Scientific, Marlborough, MA, USA) is a parachute-shaped device, ${ }^{20}$ based on a nitinol metal frame covered by a porous 160-micron polyester membrane (polyethylene terephthalate [PET]) that faces the atrial surface (Table 1). The device is self-expanding and presents 10 active fixation barbs radially positioned around the middle portion of the frame. The barbs anchor it to the LAA walls, preventing dislodgement and migration following deployment. The permeable membrane acts as a filter, catching emboli that might be generated within the pouch, and promotes endothelialisation over the device. After femoral venous access and transseptal puncture, the device is deployed via a 14-Fr sheath and a 12-Fr delivery catheter under transoesophageal echocardiography (TEE) guidance. Watchman is manufactured in five different sizes, ranging from 21 to $33 \mathrm{~mm}(3 \mathrm{~mm}$ increments). Selection is based on the largest ostial diameter of the LAA; an oversizing resulting in a 9-25\% device compression is desirable and recommended to ensure adequate stability.

The Watchman FLX was introduced in Europe in November 2015, and presented several new features compared to the previous generation. Similar to the previous generation, the Watchman FLX was a self-expanding nitinol frame structure with a polyester membrane, presenting some new features: $10-20 \%$ shorter device length, flat proximal face, atraumatic closed distal end with fluoroscopic marker, increased number of struts (18 versus 10) and barbs (12 in two rows). However, the device was withdrawn in March 2016 due to a higher than expected embolisation rate. A new version of the Watchman FLX has been developed and was released in 2018. Beginning in May 2018, a new clinical trial has been enrolling patients from several US centres aiming at assessing the safety and effectiveness of the new Watchman FLX. ${ }^{21}$ To date, the Watchman device has been the most extensively studied device for LAA occlusion.

A consistent body of clinical evidence derives from two large trials: PROTECT-AF (Watchman Left Atrial Appendage System for Embolic Protection in Patients with Atrial Fibrillation)22 and PREVAIL (Prospective Randomized Evaluation of the Watchman LAA Closure Device In Patients With Atrial Fibrillation Versus Long Term Warfarin Therapy). ${ }^{23}$ PROTECT-AF ${ }^{22}$ is a prospective, open-label, randomised trial of non-inferiority of the Watchman device versus warfarin in patients with non-valvular AF with a high stroke risk, who are eligible for 
Table 1: Main feature of the most common devices for left atrial appendage closure

\begin{tabular}{|c|c|c|c|c|c|}
\hline & Watchman & Amulet & LAmbre & WaveCrest & LARIAT \\
\hline Design & Parachute-shaped & Lobe and disk & $\begin{array}{l}\text { Cover and umbrella } \\
\text { connected via a } \\
\text { central articulating } \\
\text { waist }\end{array}$ & Umbrella-shaped & $\begin{array}{l}\text { Epicardial ligation system guided by } \\
\text { an endocardial magnetic-tipped wire }\end{array}$ \\
\hline Sizes & $\begin{array}{l}5 \text { sizes }(21,24,27,30 \text {, } \\
33 \mathrm{~mm})\end{array}$ & $\begin{array}{l}8 \text { sizes }(16,18,20,22, \\
25,28,31,34 \mathrm{~mm})\end{array}$ & $\begin{array}{l}11 \text { sizes }(16,18,20,22, \\
24,26,28,30,32,34 \\
36 \mathrm{~mm})\end{array}$ & 3 sizes $(22,27,32 \mathrm{~mm})$ & $\begin{array}{l}\text { W40 } \times \text { H20 } \times \text { L70 } \\
(\text { LARIAT+: W45) }\end{array}$ \\
\hline Sheath & $14 \mathrm{~F}$ & $12-4 \mathrm{~F}$ & $8-10 F$ & $12 \mathrm{~F}$ & $12 \mathrm{~F}$ \\
\hline
\end{tabular}

long-term VKA therapy. The primary efficacy endpoint was a composite of stroke, cardiovascular death and systemic embolism. The primary efficacy endpoint included major bleeding, pericardial effusion and device embolisation.

The trial enrolled 707 patients, who were randomised in a 2:1 fashion. Device closure of the LAA achieved non-inferiority at 18 months of follow-up with a probability of $99.9 \%$ (3.0\% per 100-patient/years in the device group versus $4.3 \%$ per 100-patient/years in the warfarin group; rate ratio [RR] $0.62,95 \%$ credible interval [Crl], 0.35-1.25). A higher rate of adverse events was reported in the device group (7.4 per 100 patient-years, 95\% Crl, 5.5-9.7, versus 4.4 per 100 patient-years, $95 \% \mathrm{Crl}, 2.5-6.7$; RR 1.69, 95\% Crl, 1.01-3.19), the most common adverse event being pericardial effusion. A $4.8 \%$ rate of pericardial effusion requiring drainage was reported in the Watchman group. ${ }^{22}$ Of note, a time-dependent distribution of safety events was observed in the trial: after a high initial rate of procedure-related complications, a reduction in adverse events was observed over time as a result of the increasing operator experience. Similar findings were confirmed in the registry that followed the trial, ${ }^{24}$ in which a large event rate that occurred during the initial procedures was followed by a progressive, significant reduction of safety events over the remaining study period.

At 4 years of follow-up, ${ }^{25} 39$ events among 463 patients (8.4\%) in the device group and 34 events among 244 patients (13.9\%) in the warfarin group were documented, meeting pre-specified criteria for both non-inferiority (posterior probability, >99.9\%) and superiority (posterior probability, 96.0\%). The Watchman implant led to a significant decrease in both cardiovascular mortality ( $\mathrm{HR}, 0.40$; $95 \% \mathrm{Cl}, 0.21-0.75 ; \mathrm{p}=0.005)$ and all-cause mortality ( $\mathrm{HR}, 0.66 ; 95 \%$ $\mathrm{Cl}, 0.45-0.98 ; \mathrm{p}=0.04)$.

The following PREVAIL trial ${ }^{23}$ enrolled 407 patients randomised to Watchman or warfarin in a 2:1 fashion. A co-primary endpoint of procedural safety was included, as a pre-defined safety criterion set by the FDA. A 2.2\% procedural complication rate was observed in the trial, which was significantly lower than the that of the PROTECT AF trial ${ }^{22}$ and allowed to meet the pre-specified performance criterion. In a recent patient-level meta-analysis of the 5-year outcomes of PROTECT AF and PREVAIL trials, ${ }^{26}$ the composite endpoint and all-stroke/systemic embolism rate were similar between groups ( $H R, 0.820 ; p=0.27$ and $H R, 0.961 ; p=0.87$, respectively). The incidence of ischaemic stroke/systemic embolism were higher in the device group; however, this difference was not statistically significant ( $\mathrm{HR}$, 1.71; $p=0.080$ ). Conversely, haemorrhagic stroke, disabling/fatal stroke, cardiovascular/unexplained death, all-cause death, and post-procedure bleeding were significantly lower with the Watchman device (haemorrhagic stroke HR, 0.20; $\mathrm{p}=0.0022$; disabling/fatal stroke HR, 0.45; $\mathrm{p}=0.03$; cardiovascular/unexplained death HR, 0.59; $\mathrm{p}=0.027$; all-cause death $\mathrm{HR}, 0.73 ; \mathrm{p}=0.035$; post-procedure bleeding $H R, 0.48 ; p=0.0003$ ).

The EWOLUTION (Registry on Watchman Outcomes in Real-Life Utilization) registry evaluated the safety and procedural success of 1,025 patients undergoing commercial LAA closure with a Watchman device. ${ }^{27}$ The registry included patients with high stroke risk (mean CHA2DS2-VASC Score $4.5 \pm 1.6$ ) and moderate-to-high bleeding risk (mean HAS-BLED [Hypertension, abnormal renal and liver function, stroke, bleeding, labile INR, elderly, drugs or alcohol] score $2.3 \pm 1.2$ ). The reported procedure success was $98.5 \%$ and the incidence of serious adverse events (SAES) within 30 days was lower than that observed in the previous randomised trials, and significantly lower for patients judged ineligible for OAC compared to those without OAC contraindications (6.5\% versus $10.2 \%$, $\mathrm{p}=0.042$ ). The most frequent SAE was major bleeding requiring blood transfusion (0.6\%). Pericardial effusion, vascular damage, procedural air embolism and device embolisation occurred in $0.2-0.4 \%$ of cases. The overall rate of stroke, TIA or systemic embolism was 1.5 per 100 patient-years, which is $84 \%$ lower than that expected on the basis of the CHA2DS2-VASC score. Data from the EWOLUTION registry and other recent registries (Table 2) 22-24,27-30 have shown a better procedural success and safety profile compared to that observed in the PROTECT AF ${ }^{22}$ and PREVAIL ${ }^{23}$ trials. Specifically, the incidence of procedure- and device-related adverse events was $2.8 \%$ in the EWOLUTION registry, ${ }_{1}^{27} .7 \%$ in the PROTECT AF trial, ${ }^{22} 4.1 \%$ in the Continued Access Protocol registry ${ }^{24}$ and $4.2 \%$ in the PREVAIL trial. ${ }^{23}$ Device deployment was successful in $98.5 \%$ of cases, with a median number of devices used of only 1.07 . Overall, $92.7 \%$ of patients needed only one device, which was properly positioned at the first attempt in $71.1 \%$ of cases. Device deployment fulfilled the criteria for LAA closure in $99.3 \%$ of cases.

Those observations suggest a significant improvement in implant techniques with better operator training over the years, which contributed to a higher success rate with lower complications.

Additionally, a cost-effectiveness analysis of LAA closure with Watchman showed that the NOACs and the Watchman implant were cost effective compared to VKAs, and the Watchman implant was cost effective relative to NOACs. ${ }^{31,32}$

\section{Amplatzer ${ }^{\mathrm{TM}}$ Cardiac Plug and Amplatzer Amulet Device}

The Amplatzerтм Cardiac Plug (Abbott Vascular, formerly St Jude Medical, St Paul, MN, USA) is a self-expanding nitinol device with a distal lobe and a proximal lobe connected by an articulated waist (Table 1). 
Table 2: Main studies on Watchman left atrial appendage device

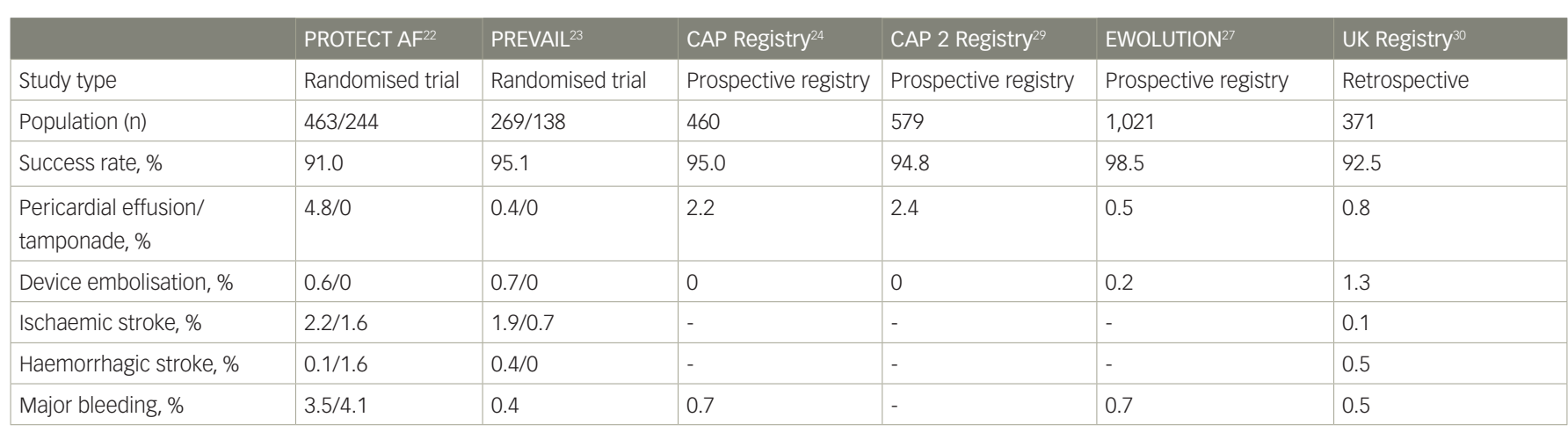

For the randomised trials, the figures represent intervention/control. Hyphens used where there is no available data.

Table 3: Main studies on the Amplatzer Cardiac Plug and Amplatzer Amulet Device

\begin{tabular}{|c|c|c|c|c|c|c|}
\hline & Tzikas et al. ${ }^{34}$ & Koskinas et al. ${ }^{36}$ & Berti et al. ${ }^{37}$ & Urena et al. ${ }^{38}$ & Lopez Minguez et al. ${ }^{39}$ & Kleinecke et al. ${ }^{40}$ \\
\hline Study type & Retrospective & Prospective & Prospective & Retrospective & Prospective & Retrospective \\
\hline Device type & $\mathrm{ACP}$ & ACP 408, Amulet 92 & ACP 91, Amulet 17 & $\mathrm{ACP}$ & $\mathrm{ACP}$ & Amulet \\
\hline Population (n) & 1,047 & 500 & 108 & 52 & 167 & 50 \\
\hline Success rate, $\%$ & 97.3 & 97.8 & 100.0 & 98.1 & 94.6 & 98.0 \\
\hline $\begin{array}{l}\text { Pericardial effusion/ } \\
\text { tamponade, \% }\end{array}$ & 1.2 & 3.2 & 2.7 & 1.9 & 1.2 & 4.0 \\
\hline Device embolisation, \% & 0.7 & 2.0 & 0 & 1.9 & - & 2.0 \\
\hline Ischaemic stroke, \% & 2.3 & - & 2.2 & 1.9 & 3.9 & 6.1 \\
\hline Haemorrhagic stroke, \% & 2 & - & 1.1 & - & - & - \\
\hline Major bleeding, \% & 1.2 & 3.2 & 0.9 & 3.8 & 5.7 & 4.0 \\
\hline
\end{tabular}

For the randomised trials, the figures represent intervention/control. Hyphens used where there is no available data. $A C P=$ Amplatzer Cardiac Plug.

The AmplatzerTM Amulet Device is the second generation of the device and presents the following improvements: eight different sizes (14-34 mm), which are suitable for LAA sizes ranging from $11 \mathrm{~mm}$ to $31 \mathrm{~mm}$, a larger proximal lobe (6-7 mm more than the distal one compared to $4-6 \mathrm{~mm}$ of the previous generation), a longer distal lobe (7.5-10.0 mm) with more stabilising wires (6-10 pairs versus 6 pairs of the ACP). Size selection is based on the largest diameter at 10-12 mm from the LAA ostium; an oversizing of $2-4 \mathrm{~mm}$ is desirable to ensure adequate stability.

The ACP and Amplatzer Amulet devices received CE Mark approval in 2008 and 2013, respectively, but are not available for commercial use in the USA yet. However, the ongoing international multicentre randomised Amulet investigational device exemption trial will randomise approximately 1,800 patients in a 1:1 fashion to either Amplatzer Amulet or Watchman over a 5-year follow-up period. ${ }^{33}$

A recent multicentre experience with the ACP involved 22 European centres and 1,047 patients, reporting a high rate of procedural success (97.0\%), with a $4.9 \%$ rate of periprocedural adverse events. ${ }^{34}$ The global, prospective Amplatzer Amulet observational study is a real-world registry enrolling a large cohort $(n=1,088)$ of patients who received the Amplatzer Amulet device. ${ }^{35}$ Successful implantation was reported in $99.0 \%$ of patients, whereas major adverse events occurred in 3.2\% of cases during the procedure and the index hospitalisation. An effective LAA closure, represented by the absence of a significant $(\geq 3 \mathrm{~mm}$ ) peri-device leak, was documented in $98.2 \%$ of patients via TEE. Major findings from other registries are reported in Table 3.34,36-40

\section{Other endocardial/percutaneous devices}

The LAmbreTM LAA closure system (Lifetech Scientific Co, Ltd, Shenzhen, China) is a self-expanding, nitinol-based device, which obtained the CE mark in June 2016 (Table 1). The device presents a proximal, PET-filled cover, connected via a central, articulating waist to a distal umbrella presenting eight claws and an additional PET cover. Fifteen different sizes are available (16-36 mm), as well as two different designs based on the cover size $(4-6 \mathrm{~mm}$ or 12-14 $\mathrm{mm}$ larger than the umbrella). On the basis of the measured LAA orifice, an oversizing of 4-8 $\mathrm{mm}$ is suggested to achieve adequate stability. A recent prospective, multicentre experience reported procedural and follow-up data from 153 patients who received LAmbre implantation in 12 Chinese centres. ${ }^{41}$ The authors reported a successful deployment in $99.4 \%$ of patients, $3.3 \%$ periprocedural major adverse events, and the absence of a significant $(\geq 3 \mathrm{~mm}$ ) residual peri-device leak in $99 \%$ of patients after 12 months of follow-up.

The WaveCrest ${ }^{\circledR}$ LAA occlusion system (Biosense Webster, Inc., [a Johnson and Johnson company], Irwindale, CA, USA) is a single lobe device with an umbrella shape (Table 1), covered by expanded polytetrafluoroethylene (ePTFE). Three sizes are currently available and 20 circumferentially distributed micro-tines are present at the distal margin to ensure anchorage. Preliminary data from the Coherex WAVECREST I Left Atrial Appendage Occlusion Study showed a 2.7\% periprocedural complication rate and a primary efficacy of $92 \%$ for the intention-to-treat protocol and $97 \%$ for the as-treated protocol. ${ }^{42}$ 
Table 4: Main studies on the LARIAT device

\begin{tabular}{|l|l|l|l|}
\hline & Lakkireddy et al. ${ }^{45}$ & Bartus et al. $^{46}$ & Price et al. $^{47}$ \\
\hline Study type & Prospective & Retrospective & Retrospective \\
\hline Population (n) & 712 & 89 & 154 \\
\hline Success rate, \% & 98 & 96 & 94 \\
\hline $\begin{array}{l}\text { Pericardial effusion/ } \\
\text { tamponade, \% }\end{array}$ & - & - & 10.4 \\
\hline Ischaemic stroke, \% & - & & - \\
\hline Haemorrhagic stroke, \% & - & - & 1.1 \\
\hline Major bleeding, \% & 0 & 1.1 & - \\
\hline
\end{tabular}

For the randomised trials, the figures represent intervention/control.

The Ultraseal LAA closure device (Cardia, Inc., Eagan, MN, USA) is a self-expandable device that consists of a proximal sail, a distal bulb with 12 hooks and an articulating joint that connects previous sections and promotes high device conformability to different LAA morphologies. There are nine available sizes (16-32 $\mathrm{mm})$, the seal being $6 \mathrm{~mm}$ larger than the distal bulb. Size selection is based on the largest diameter at 10-12 mm from the LAA ostium; an oversizing of $25-33 \%$ of the bulb is desirable to ensure adequate stability. Two small studies reported a high success rate without any major periprocedural complications. ${ }^{43,44}$

The LARIAT ${ }^{\circledR}$ system (SentreHEART, Redwood City, CA, USA) is a hybrid percutaneous/epicardial device (Table 1), consisting of a pre-tied suture loop guided by an endocardial magnetic-tipped wire delivered over the LAA. This device should be considered in patients with absolute contraindication to OAC and/or antiplatelet therapy, since there is no need for further antithrombotic therapy after implantation. This system also should be considered in patients with a very large LAA (the upper limit of the LARIAT is $40 \mathrm{~mm}$, larger than any other available device). Additionally, the suture can achieve LAA electrical isolation; therefore the $\mathrm{AF}$, in addition to stroke prevention, is potentially reduced.

The LARIAT system has been investigated only in small studies (Table 4). ${ }^{45-7}$ The larges study on the LARIAT system reported a success rate $>95 \%$ and a procedure-related mortality of $0.14 \%$. Further studies and, in particular, randomised, controlled trials are necessary to assess the safety profile and efficacy in the stroke prevention of this implant. A list of all the ongoing registered clinical trials on LAA closure is reported in Table 5.

\section{Oral anticoagulation following left atrial appendage closure}

The proposed antithrombotic regimen after Watchman device implantation is extremely contorted. It consists of 6 weeks of warfarin (international normalised ratio 2.0-3.0) plus aspirin (81-100 mg/day), followed by aspirin (up to $300-325 \mathrm{mg} /$ day) plus clopidogrel $(75 \mathrm{mg} /$ day) for 6 months if follow-up TEE shows no significant peri-device leaks. After 6 months, patients can switch to aspirin alone at a dosage of 300-325 mg/day.

If TEE at 6 weeks shows incomplete occlusion of the LAA, the patient should continue OAC and repeat TEE after 6 weeks.

According to the findings of the ASAP trial (ASA Plavix Feasibility Study with WATCHMAN Left Atrial Appendage Closure Technology), LAA closure with the Watchman device can be safely performed without the 6-week warfarin bridging; dual antiplatelet therapy with aspirin and
6 months of clopidogrel has proven to be a safe alternative to the 45-day warfarin regimen. ${ }^{48}$ The EWOLUTION registry supported these results by demonstrating no significant difference in rates of stroke, thrombus formation on device or major bleeding in patients treated with dual antiplatelet therapy compared to warfarin alone or NOACs following Watchman implantation. ${ }^{27}$ Given the low thrombogenic profile of the ACP device, 6 months of post-procedural dual antiplatelet therapy appears reasonable. ${ }^{49}$

\section{Left atrial appendage closure-related issues}

Device-related thrombus (DRT) and incomplete LAA closure resulting in a significant leak represent the two main limitations/complications after percutaneous LAA closure. In a recent report on 344 patients with an $A C P$, DRT was observed in $3.2 \%$ and was not associated with an increased risk of cardiovascular events..$^{50}$ Independent predictors of DRT were smoking (OR, 5.79; $\mathrm{p}=0.017)$ and female sex (OR, 4.22; $\mathrm{p}=0.027)$.

However, in a larger series of 1,739 Watchman patients, ${ }^{51}$ the prevalence of DRT was $3.74 \%$ and the rate of stroke and system thromboembolism was significantly higher among those with a documented DRT (7.46 and 1.78 per 100 patient-years; adjusted RR, 3.55; 95\% Cl, 2.18-5.79; $p<0.001$ ).

Clopidogrel is used after a Watchman implant to prevent DRT, which might occur before endothelialisation is complete. However, loss-of-function polymorphisms of the cytochrome P450 2C19 (CYP2C19) gene are associated with reduced hepatic bioactivation of clopidogrel and may lead to potential consequences. In our practice, all Watchman patients are genotyped for CYP2C19 polymorphisms with an assay using a polymerase chain reaction with allele-specific primer extension. Patients are classified as having normal, reduced or increased clopidogrel metabolism. Patients with reduced metabolism are considered resistant to clopidogrel and, if no contraindications exist, prasugrel or a half-dose of a novel OAC during the dual antiplatelet therapy (DAPT) phase following Watchman FLX implant is prescribed.

Another drawback associated with LAA procedure is the presence of incomplete LAA closure resulting in a significant leak. A residual LAA patency after endocardial procedures generally results from incomplete contact between the plug and the LAA wall; therefore, leaks can be observed at the edge of the device. Conversely, leaks after LARIAT procedures are centrally located, as a result of incomplete suture tightening and/or tissue necrosis. Whether or not incomplete LAA closure is associated with an increased risk of thromboembolic events is still unclear. An association between incomplete LAA closure and stroke/systemic embolisation was observed after surgical ligation.52

Similar findings were not confirmed in patients with percutaneous LAA closure. ${ }^{50,53}$ However, the rate of thromboembolic events was considerably low in these studies, potentially jeopardising the overall power of the results. An additional limitation is represented by the imaging technique utilised to assess the presence of leaks. Two-dimensional TEE is generally the strategy of choice to assess for a persistent LAA patency during follow-up. However, several reports have confirmed the inferiority of this technique compared to three-dimensional TEE or computerised tomography, which may potentially underestimate the presence and size of residual leaks.

Nevertheless, it is common practice not to discontinue OAC in patients with leaks $\geq 5 \mathrm{~mm}$, unless contraindicated. Further studies are necessary to assess the clinical significance of incomplete LAA closure. 
Table 5: Ongoing registered clinical trials on left atrial appendage closure

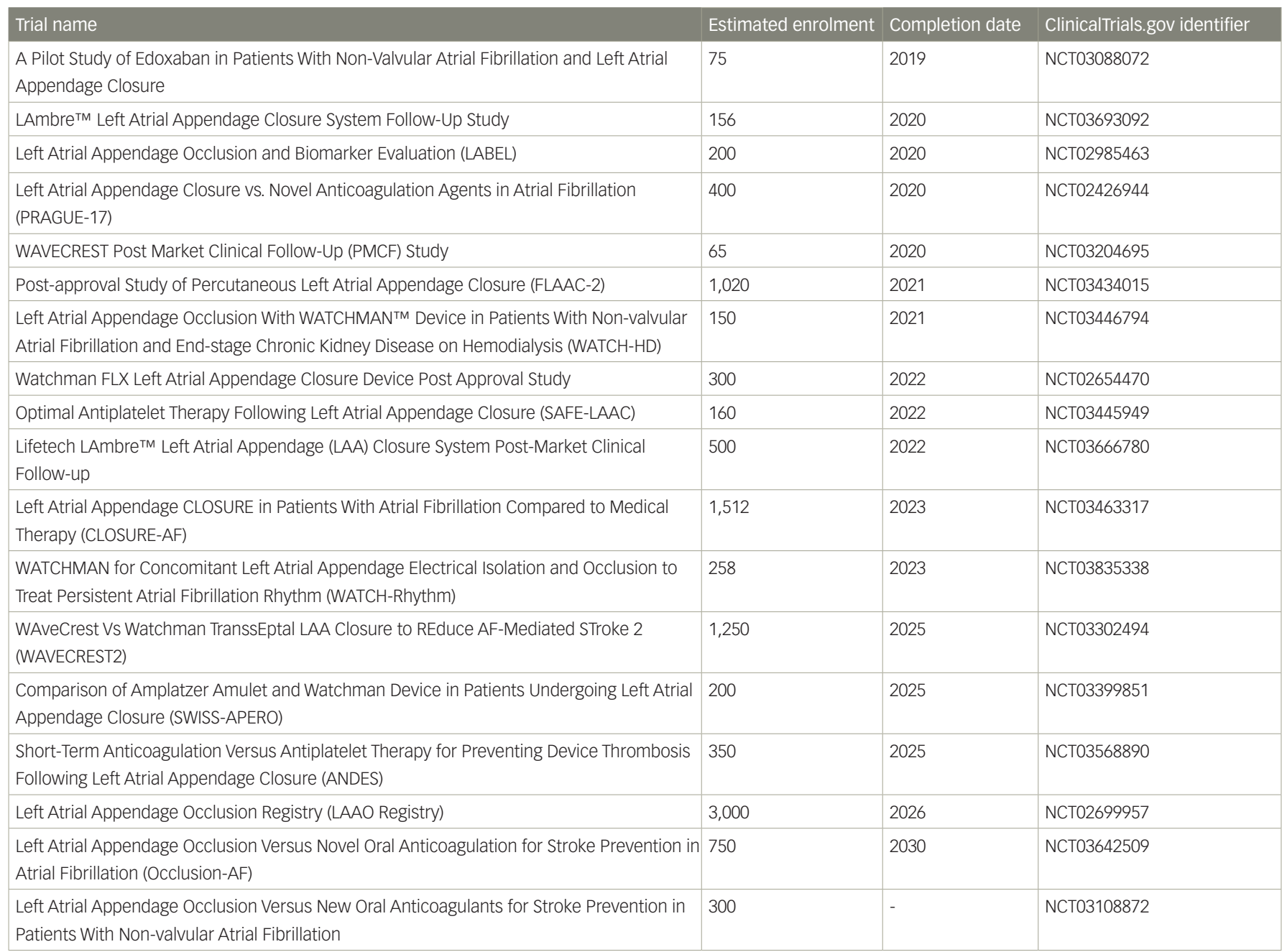

Source: Clinicaltrials.gov

\section{Conclusions}

Percutaneous LAA closure has become a safe and effective alternative to OAC in patients with non-valvular AF who have a high-risk for stroke. Results from clinical trials and real-world registries enrolling patients with a Watchman or an Amplatzer device showed a high success rate, a low rate of major complications and an effective prevention of thromboembolic and major haemorrhagic events.

Further studies are needed to optimise device and patient selection and to improve anticoagulation management after LAA closure. $\square$
1. January CT, Wann LS, Calkins H, et al. 2019 AHA/ACC/HRS focused update of the 2014 AHA/ACC/HRS guideline for the management of patients with atrial fibrillation: a report of the American College of Cardiology/American Heart Association Task Force on Clinical Practice Guidelines and the Heart Rhythm Society. J Am Coll Cardiol. 2019;74:104-32.

2. Chugh SS, Havmoeller R, Narayanan K, et al. Worldwide epidemiology of atrial fibrillation: a Global Burden of Disease 2010 Study. Circulation. 2014;129:837-47.

3. Heeringa J, van der Kuip DA, Hofman A, et al. Prevalence, incidence and lifetime risk of atrial fibrillation: the Rotterdam study. Eur Heart J. 2006;27:949-53.

4. Benjamin EJ, Wolf PA, D'Agostino RB, et al. Impact of atrial fibrillation on the risk of death: the Framingham Heart Study. Circulation. 1998:98:946-52.

5. Vidaillet $\mathrm{H}$, Granada JF, Chyou $\mathrm{PH}$, et al. A population-based study of mortality among patients with atrial fibrillation or flutter. Am J Med. 2002:113:365-70.

6. Wolf PA, Abbott RD, Kannel WB. Atrial fibrillation as an independent risk factor for stroke: the Framingham Study. Stroke. 1991;22:983-8.

7. Lin HJ, Wolf PA, Kelly-Hayes M, et al. Stroke severity in atrial fibrillation: the Framingham Study. Stroke. 1996;27:1760-4.

8. Benjamin EJ, Virani SS, Callaway CW, et al. Heart disease and stroke statistics-2018 update: a report from the American Heart Association. Circulation. 2018;137:e67-e492.

9. Coyne KS, Paramore C, Grandy S, et al. Assessing the direct costs of treating nonvalvular atrial fibrillation in the United States. value Health. 2006;9:348-56.
10. Blackshear J, Odell JA. Appendage obliteration to reduce stroke in cardiac surgical patients with atrial fibrillation. Ann Thorac Surg. 1996;61:755-9.

11. Di Biase L, Burkhardt JD, Mohanty P, et al. Left atrial appendage: an underrecognized trigger site of atrial fibrillation. Circulation 2010;122:109-18

12. Pollick C, Taylor D. Assessment of left atrial appendage function by transesophageal echocardiography. Implications for the development of thrombus. Circulation. 1991;84:223-31.

13. Nucifora G, Faletra FF, Regoli F, et al. Evaluation of the left atrial appendage with real-time 3-dimensional transesophageal echocardiography: implications for catheter-based left atria appendage closure. Circ Cardiovasc Imaging. 2011:4:514-23.

14. Di Biase L, Santangeli P, Anselmino M, et al. Does the left atrial in L, Santan appendage morphology correlate with the risk of stroke in
patients with atrial fibrillation? Results from a multicenter study. patients with atrial fibrillation? Res
J Am Coll Cardiol. 2012;60:531-8.

15. Hart RG, Pearce LA, Aguilar MI. Meta-analysis: antithrombotic therapy to prevent stroke in patients who have nonvalvular atrial fibrillation. Ann Intern Med. 2007;146:857-67

16. Gomes T, Mamdani MM, Holbrook AM, et al. Rates of hemorrhage during warfarin therapy for atrial fibrillation. CMAJ. 2013;185:E121-7.

17. O'Brien EC, Holmes DN, Ansell JE, et al. Physician practices regarding contraindications to oral anticoagulation in atrial fibrillation: findings from the Outcomes Registry for Better Informed Treatment of Atrial Fibrillation (ORBIT-AF) registry. Am Heart J. 2014;167:601-9.

18. Ruff CT, Giugliano RP, Braunwald E, et al. Comparison of the efficacy and safety of new oral anticoagulants with warfarin in patients with atrial fibrillation: a meta-analysis of randomized trials. Lancet. 2014:383:955-62.

19. Kakkar AK, Mueller I, Bassand JP, et al. GARFIELD Registry Investigators. Risk profiles and antithrombotic treatment of patients newly diagnosed with atrial fibrillation at risk of stroke perspectives from the international, observational, prospective GARFIELD registry. PLoS One. 2013;8:e63479.

20. Kramer DB, Kesselheim AS. The Watchman saga--closure at last? N Eng/J Med. 2015;372:994-5.

21. Investigational Device Evaluation of the WATCHMAN FLXTM LAA Closure Technology (PINNACLE FLX). Available at: https://clinicaltrials.gov/ct2/show/study/NCT02702271 (accessed 14 May 2019) ClinicalTrials.gov Identifier NCT02702271.

22. Holmes DR, Reddy VY, Turi ZG, et al. PROTECT AF Investigators Percutaneous closure of the left atrial appendage versus warfarin therapy for prevention of stroke in patients with atrial fibrillation: a randomised non-inferiority trial. with atrial fibrillation: a rando

23. Holmes DR Jr, Kar S, Price MJ, et al. Prospective randomized evaluation of the Watchman Left Atrial Appendage Closure device in patients with atrial fibrillation versus long-term warfarin therapy: the PREVAlL trial. J Am Coll Cardiol. 2014;64:1-12.

24. Reddy VY, Holmes D, Doshi SK, et al. Safety of percutaneous left atrial appendage closure: results from the Watchman Left Atrial Appendage System for Embolic Protection in Patients with AF (PROTECT AF) clinical trial and the Continued Access Registry. Circulation. 2011;123:417-24.

25. Reddy VY, Sievert $\mathrm{H}$, Halperin J, et al. Percutaneous left 
atrial appendage closure vs warfarin for atrial fibrillation: a randomized clinical trial. JAMA. 2014;312:1988-98.

26. Reddy VY, Doshi SK, Kar S, et al. 5-Year outcomes after left atrial appendage closure: from the PREVAIL and PROTECT AF trials. J Am coll Cardiol 2017:70:2964-75.

27. Boersma LV, Schmidt B, Betts TR, et al. EWOLUTION investigators. Implant success and safety of left atrial appendage closure with the WATCHMAN device: peri-procedural outcomes from the EWOLUTION registry. Eur Heart J. 2016;37:2465-74.

28. Holmes DR, Jr, Doshi SK, Kar S, et al. Left atrial appendage closure as an alternative to warfarin for stroke prevention in atrial fibrillation: a patient-level meta-analysis. J Am Coll Cardiol. 2015;65:2614-23.

29. Betts TR, Leo M, Panikker S, et al. Percutaneous left atrial appendage occlusion using different technologies in the United Kingdom: a multicenter registry Catheter Cardiovasc Interv. 2017;89:484-92.

30. Reddy VY, Gibson DN, Kar S, et al. Post-approval U.S. experience with left atrial appendage closure for stroke prevention in atrial with left atrial appendage closure for stroke p
fibrillation. J Am Coll Cardiol. 2017;69:253-61.

31. Reddy VY, Akehurst RL, Armstrong SO, et al. Cost effectiveness of left atrial appendage closure with the Watchman device for atrial fibrillation patients with absolute contraindications to warfarin. Europace. 2016:18:979-86.

32. Reddy VY, Akehurst RL, Armstrong SO, et al. Time to cost-effectiveness following stroke reduction strategies in $\mathrm{AF}$ : warfarin versus NOACs versus LAA closure. J Am Coll Cardio. 2015;66:2728-39

33. AMPLATZERTM Amulet'TM LAA Occluder Trial (Amulet IDE). https://clinicaltrials.gov/ct2/show/NCT02879448 (accessed 15 May 2019). ClinicalTrials.gov Identifier: NCT02879448.

34. Tzikas A, Shakir S, Gafoor S, et al. Left atrial appendage occlusion for stroke prevention in atrial fibrillation: multicentre experience with the AMPLATZER Cardiac Plug. Eurolntervention. 2016:11:1170-9.
35. Landmesser U, Schmidt B, Nielsen-Kudsk JE, et al. Left atrial appendage occlusion with the AMPLATZER Amulet device: periprocedura penproceducl and early clinical/chocardlographic data from a global prospective

36. Koskinas KC, Shakir S, Fankhauser M, et al. Predictors of early (1-week) outcomes following left atrial appendage closure with (1-week) outcomes following left atrial appendage closure

37. Berti S, Pastormerlo LE, Rezzaghi M, et al. Left atrial appendage occlusion in high-risk patients with non-valvular atrial fibrillation. Heart. 2016;102:1969-73.

38. Urena M, Rodés-Cabau J, Freixa X, et al. Percutaneous left atria appendage closure with the AMPLATZER cardiac plug device in patients with nonvalvular atrial fibrillation and contraindications to anticoagulation therapy. J Am Coll Cardiol. 2013;62:96-102.

39. López Mínguez JR, Asensio JM, Gragera JE, et al. Two-year clinical outcome from the Iberian registry patients after left atrial appendage closure. Heart. 2015;101:877-83.

40. Kleinecke C, Park JW, Gödde M, et al. Twelve-month follow-up of left atrial appendage occlusion with Amplatzer Amulet. Cardio left atrial append $24: 131-8$

41. Huang $\mathrm{H}, \mathrm{Li}$ Y $Y$, Xu Y, et al. Percutaneous left atrial appendage closure with the LAmbre device for stroke prevention in atrial fibrillation: a prospective, multicenter clinical study. JACC Cardiovasc Interv. 2017;10:2188-94.

42. Coherex WAVECREST I Left Atrial Appendage Occlusion Study. https://clinicaltrials.gov/ct2/show/NCT02239887 (accessed 15 May 2019). ClinicalTrials.gov Identifier. NCT02239887.

43. Regueiro A, Bernier M, O'Hara G, et al. Left atrial appendage closure: Initial experience with the ultraseal device. Catheter Cardiovasc Interv. 2017;90:817-23.

44. Sabiniewicz R, Hiczkiewicz J, Wańczura P, et al. First-in-human experience with the Cardia Ultraseal left atrial appendage closure device: The feasibility study. Cardiol J. 2016;23:652-4.

45. Lakkireddy D, Afzal MR, Lee RJ, et al. Short and long-term outcomes of percutaneous left atrial appendage suture ligation: results from a US multicenter evaluation. Heart Rhythm 2016;13:1030-6

46. Bartus K Han FT, Bednarek $\rfloor$ et al. Percutaneous left atrial appendage suture ligation using the LARIAT device in patients with atrial fibrillation: initial clinical experience I Am Coll Cardio 2013;62:108-18.

47. Price MJ, Gibson DN, Yakubov SJ, et al. Early safety and efficacy of percutaneous left atrial appendage suture ligation: results from the U.S. transcatheter LAA ligation consortium. J Am Coll Cardiol. 2014;64:565-72

48. Reddy VY, Möbius-Winkler S, Miller MA, et al. Left atrial appendage closure with the Watchman device in patients with a contraindication for oral anticoagulation: the ASAP study (ASA Plavix Feasibility Study with Watchman Left Atrial Appendage Closure Technology). J Am Coll Cardiol. 2013;61:2551-6.

49. Parks J, Bethencourt $A$, Sievert $H$, et al. Left atrial appendage closure with Amplatzer cardiac plug in atrial fibrillation: initi 2011;77:700-6.

50. Saw J, Tzikas A, Shakir S, et al. Incidence and clinical impact of device-associated thrombus and peri-device leak following left atrial appendage closure with the Amplatzer Cardiac Plug. JACC Cardiovasc Interv. 2017:10:391-9.

51. Dukkipati SR, Kar S, Holmes DR, et al. Device-related thrombus after left atrial appendage closure. Circulation. 2018;138:874-85. 52. Aryana A, Singh SK, Singh SM, et al. Association between incomplete surgical ligation of left atrial appendage and stroke and systemic embolization. Heart Rhythm. 2015;12:1431-7.

53. Viles-Gonzalez JF, Kar S, Douglas $\mathrm{P}$, et al. The clinical impact of incomplete left atrial appendage closure with the Watchman Device in patients with atrial fibrillation: a PROTECT AF (Percutaneous Closure of the Left Atrial Appendage Versus Warfarin Therapy for Prevention of Stroke in Patients With Atrial Fibrillation) substudy. J Am Coll Cardiol. 2012:59:923-9. 